\title{
Sobrepeso e obesidade em escolares pré-púberes: associação com baixo peso ao nascer e antecedentes familiares para doença cardiovascular. Embu - Região Metropolitana de São Paulo, 2006.
}

\author{
Overweight and obesity in prepubertal schoolchildren: \\ the association with low birth weight and family antecedents \\ of cardiovascular disease. Embu - Metropolitan Region \\ of São Paulo, 2006.
}

\footnotetext{
Maria Wany Louzada Strufaldi ${ }^{1}$

Edina Mariko Koga da Silva ${ }^{2}$

Rosana Fiorini Puccini ${ }^{1}$
}

${ }^{1}$ Pediatria Geral e

Comunitária do

Departamento de Pediatria,

Universidade Federal de São

Paulo. Rua Botucatu 598,

Vila Clementino.

04023-062 São Paulo SP.

rpuccini@terra.com.br

${ }^{2}$ Departamento de

Medicina, Universidade

Federal de São Paulo

\begin{abstract}
The aim was to determine the prevalence of overweight and obesity in schoolchildren and the association with birth weight and family antecedents of cardiovascular disease. This crosssectional study used a probabilistic sample of 929 schoolchildren aged 6 to 10 years. The variables were: body mass index (BMI), birth weight and family antecedents of cardiovascular disease. The statistical analysis consisted of the chi-square test (Pearson) and odds ratio, as association measurements. Of the schoolchildren (54.6\% of which were female), $14.4 \%$ and $13.3 \%$ were overweight and obese, respectively. Low birth weight was reported among $9.4 \%$ and family antecedents of cardiovascular disease among 35.2\%. Overweight or obesity (BMI e" P85) was associated with the presence of family antecedents of cardiovascular disease (OR = 1.66; 95\% CI 1.23-2.23) and male sex (OR = 1.37; 95\%CI 1.02-1.83); there was no association with birth weight. The results indicate the need for preventive actions for children with family antecedents of cardiovascular disease.
\end{abstract}

Key words: Overweight, Obesity, Children, Prevalence, Cardiovascular disease
Resumo O objetivo foi determinar a prevalência de sobrepeso e obesidade em escolares e a associação com baixo peso ao nascer e antecedentes familiares para doença cardiovascular. Estudo transversal que utilizou amostra probabilística de 929 escolares com idades de 6 a 10 anos. Variáveis: Índice de Massa Corpórea (IMC), peso ao nascer e antecedentes familiares para doença cardiovascular. Análise estatística: teste qui-quadrado (Pearson) e odds ratio como medidas de associação. $14,4 \%$ e $13,3 \%$ dos escolares $(54,6 \%$ do sexo feminino) apresentaram sobrepeso e obesidade, respectivamente. Baixo peso ao nascer foi referido em 9,4\% das crianças e a presença de antecedentes familiares para doença cardiovascular em $35,2 \%$. Sobrepeso ou obesidade (IMC maior ou igual a P85) associaram-se à presença de antecedentes familiares para doença cardiovascular $(O R=1,66$; IC95\% 1,23-2,23) e ao sexo masculino $(O R=1,37$; IC 95\% 1,02-1,83); não houve associação com o peso ao nascer. Os resultados indicam a necessidade de ações preventivas direcionadas às crianças com antecedentes familiares para doença cardiovascular.

Palavras-chave Sobrepeso, Obesidade, Crianças, Prevalência; Doença cardiovascular 


\section{Introdução}

Estimativas da Organização Mundial de Saúde (OMS), dos anos 1990-2000 apontam para um declínio na prevalência mundial de desnutrição em crianças pré-escolares de $34 \%$ para $27 \%$ segundo índice Estatura/Idade (stunting) e de $27 \%$ para 22\% segundo Peso/Estatura (wasting), sendo que na América Latina, o índice Estatura/Idade, em 2005, foi estimado em 9,6\% ${ }^{1}$. No Brasil esses índices em 2006 foram ainda menores, sendo descritos déficits de Peso/Estatura e Estatura/ Idade de 1,9\% e 7\%, respectivamente, em crianças menores de cinco anos de idade ${ }^{2}$. Por outro lado, há uma tendência de ascensão das prevalências de sobrepeso e de obesidade em crianças, tanto nos países desenvolvidos, quanto naqueles em desenvolvimento. Esse aumento na obesidade tornou-se um importante problema de saúde pública, embora aspectos singulares da transição nutricional ocorrida neste século sejam encontrados em cada país e região do mundo ${ }^{3-6}$.

No Brasil, essa transição tem ocorrido de forma heterogênea na dependência da região geográfica, classe social, faixa etária, sexo e está especialmente relacionada às áreas urbanas e à região centro-sul do país ${ }^{7-9}$. A evolução do estado nutricional segundo o Índice de Massa Corpórea (IMC) avaliada em três inquéritos nacionais entre 1974/1975 e 2002/2003 demonstra que a obesidade triplicou nos adultos do sexo masculino e, nos adultos do sexo feminino, manteve-se estável a partir de 1989. Nos estratos de renda mais pobre a obesidade aumentou significantemente em todo o período, enquanto nas faixas de renda mais elevada, a prevalência se elevou até 1989, apresentando posteriormente tendência à redução ${ }^{10,11}$. Segundo o IBGE ${ }^{11}$, entre meninos adolescentes há um aumento contínuo no excesso de peso em todos os estratos de renda nesse período; entre meninas, a frequência do excesso de peso mantémse para as mais pobres, porém com discreta redução entre as meninas de renda mais elevada entre 1989 e 2002/2003. Em pré-púberes, a prevalência de obesidade descrita em diferentes estudos no início dos anos 2000, utilizando-se o IMC como critério, é de aproximadamente $10 \%{ }^{12-14}$.

A relevância dessa nova condição nutricional em crianças e adolescentes se deve ao fato de que o excesso de peso e suas principais comorbida$\operatorname{des}^{15-18}$ - dislipidemia, hipertensão arterial, diabetes mellitus e síndrome metabólica - podem amplificar os fatores de risco para ocorrência de doença cardiovascular (DCV) no adulto. Com essa mesma preocupação, ou seja, a identificação de fatores de risco na infância para a ocorrência de DCV no adulto, incluem-se os estudos sobre o papel do peso ao nascer. Tem sido descrito que indivíduos que apresentaram baixo peso ao nascer e que desenvolvem obesidade durante a infância ou na vida adulta têm risco aumentado de apresentar DCV e resistência à insulina ${ }^{19-22}$. Não estão elucidados, entretanto, o momento e com qual intensidade ocorreriam as manifestações clínicas resultantes da interação entre os mecanismos genéticos que influenciam o peso ao nascer e a presença de fatores ambientais desencadeando obesidade, hipertensão arterial e resistência à insulina que resultariam na síndrome metabólica e, consequentemente, maior risco de DCV.

Embora o aumento da prevalência de excesso de peso em crianças e adolescentes no mundo e no Brasil, mais especificamente, já esteja bem comprovado, assim como há evidências de associação do baixo peso ao nascer com as doenças no adulto, poucos trabalhos têm abordado faixas etárias mais precoces, sobretudo escolares prépúberes, permanecendo ainda em aberto as inter-relações de fatores de risco nesse período da vida. Conhecer o estado nutricional de crianças escolares pré-púberes e possíveis associações com os antecedentes familiares de DCV e peso ao nascer é fundamental para melhor compreender esse processo, tornando possível, também, a identificação de um grupo populacional que necessita de atenção diferenciada e oportuna. O presente estudo teve como objetivo conhecer a prevalência de sobrepeso e obesidade e fatores associados em escolares pré-púberes no município do Embu, região metropolitana de São Paulo.

\section{Métodos}

Tipo de estudo: transversal, analítico.

Local: O município de Embu está situado na região metropolitana de São Paulo e, em 2006, sua população era de 244.642 habitantes. Nesse mesmo ano, o Índice de Desenvolvimento $\mathrm{Hu}$ mano (IDH) do município $(0,772)$ foi inferior à média do Estado de São Paulo $(0,841)$ e do Brasil $(0,792)$ e a taxa de mortalidade infantil foi de 11,0 por mil nascidos vivos ${ }^{23}$. A rede de saúde municipal está constituída por 13 unidades básicas de saúde, dois prontos-socorros, uma maternidade, ambulatório de especialidades, um hospital regional de 250 leitos para referência nas áreas gerais. Em conjunto com as secretarias municipais de Saúde e de Educação, a Universidade Federal de São Paulo tem desenvolvido atuação in- 
tersetorial, com destaque ao Programa de Saúde Escolar, implantado em 1984, e ao Programa Escola Promotora de Saúde, implantado em 2002.

População do estudo e amostragem: As escolas públicas atendem a 63.333 alunos no município (98\% dos estudantes matriculados no Ensino Fundamental e Médio). Em 2006, 17.869 crianças estavam matriculadas nas $1^{\text {a }}$ a $4^{\text {a }}$ série do ensino fundamental na rede pública. Foi realizada amostragem por conglomerados com sorteio das escolas em dois estágios com probabilidade proporcional ao número de alunos por escola, utilizando-se como base uma população aproximada de 18 mil escolares matriculados nessas séries e prevalência estimada de $10 \%$ de excesso de peso (IMC maior ou igual a P85). Para um intervalo de confiança de $95 \%$ e uma variação de $2 \%$ em torno da prevalência ( $8 \%$ a $12 \%$ ), o valor calculado da amostra foi de 860 crianças, cálculo realizado no programa Epinfo $6.01^{24}$. Contando com possíveis perdas e recusas de participação, definiu-se uma amostra de 1100 escolares; destes, 929 $(84,5 \%)$ crianças de 6 a 10 anos de idade retornaram com consentimento para participação no estudo com preenchimento do questionário pela família e realização da antropometria.

\section{Instrumentos e procedimentos do estudo}

Questionário respondido pelos pais das crianças da amostra, para obtenção de informações referentes aos antecedentes pessoais da criança e familiares; 2) avaliação do peso e estatura. Os escolares foram pesados em balança do tipo plataforma, digital, com capacidade de até 150 quilos. A medida de peso foi tomada com os escolares vestindo roupas leves, descalços, permanecendo eretos no centro da balança, com os braços esticados ao longo do corpo. A estatura foi medida utilizando-se fita métrica, milimetrada, presa à parede e com esquadro de madeira com trava; a medida foi efetuada com as crianças em posição ereta, descalças, calcanhares unidos, braços ao longo do corpo e a cabeça posicionada paralela ao solo.

\section{Variáveis}

1) Peso ao nascer - utilizou-se a classificação da Organização Mundial da Saúde (baixo peso, peso insuficiente, peso adequado e macrossomia $)^{25}$. Esta informação foi obtida por meio do questionário respondido pelos pais.

2) Antecedentes familiares - foram considerados antecedentes positivos quando os pais re- latavam diagnóstico de diabetes, hipertensão arterial, obesidade ou dislipidemia. Como os pais das crianças, em sua maioria, são muito jovens para apresentarem doença coronariana, foi considerado, também, como antecedente familiar positivo para DCV o relato de infarto do miocárdio ou morte súbita nos avós, ocorrida antes dos 55 anos de idade ${ }^{26}$. Esta informação foi obtida por meio de questionário respondido pelos pais.

3) Índice de massa corpórea (IMC) - expresso em $\mathrm{Kg} / \mathrm{m}^{2}$. Utilizou-se como referência a curva do CDC-NCHS (2000), que define a criança com IMC abaixo do P5 como desnutrida ou baixo peso, com IMC entre P5 e P84, como peso normal, IMC entre P85 e P94, como sobrepeso e IMC maior ou igual ao valor do percentil 95, como obesas, conforme idade e sexo ${ }^{27-29}$.

\section{Análise Estatística}

Para a comparação de variáveis categóricas utilizou-se o teste qui-quadrado, quando a análise considerou as quatro categorias de IMC como desfecho (baixo peso, IMC normal, sobrepeso e obesidade); o odds ratio foi calculado, considerando como desfecho sobrepeso/obesidade (IMC maior ou igual a P85) comparandose com IMC normal (IMC entre P5 e P84), excluindo-se crianças com IMC menor que P5. Em todos testes estatísticos adotou-se o nível de significância de 5\% $(a=0,05)$. As análises foram elaboradas com o apoio dos programas Excell 2003 e SPSS 13.0.

Este estudo foi aprovado pelo Comitê de Ética em Pesquisa da Unifesp, pelas Secretarias da Saúde e da Educação do Embu e todos os pais assinaram termo de consentimento livre e esclarecido.

\section{Resultados}

Dentre as 929 crianças incluídas no estudo, 54,6\% eram do sexo feminino; a mediana de idade foi de: 8,39 anos. Na Tabela 1 é apresentada a distribuição do estado nutricional por faixa etária. Não foi verificada diferença estatisticamente significante $(p=0,168)$ entre estado nutricional e faixa etária. A prevalência de sobrepeso e obesidade foi de $14,4 \%$ e $13,3 \%$, respectivamente; ressaltase que dentre as crianças com 6 a 7 anos, 32,9\% das crianças já apresentavam sobrepeso ou obesidade. $\mathrm{O}$ baixo peso (IMC $<\mathrm{P} 5$ ) e a baixa estatura $(\mathrm{P} / \mathrm{E}<\mathrm{P} 3)$ ocorreram em $4,1 \%$ e $5,4 \%$ das crianças, respectivamente. 
Em 328 (35,3\%) escolares participantes do estudo houve relato de antecedente familiar positivo para DCV; destes, $20 \%$ apresentavam antecedentes de DCV precoce, $17,3 \%$ de hipertensão arterial, $8,5 \%$ obesidade, $5,9 \%$ dislipidemia e $3,7 \%$ diabetes mellitus tipo 2 .

A Tabela 2 apresenta a distribuição das quatro categorias do estado nutricional segundo sexo, antecedentes familiares para DCV e peso ao nascer. Houve associação estatisticamente significante em relação ao sexo e à presença de antecedentes familiares para DCV. Verificou-se maior frequência de crianças obesas e com sobrepeso entre os meninos e com presença de antecedente familiar para DCV. A análise de cada um dos antecedentes familiares, isoladamente, não se associou ao sobrepeso/obesidade, somente quando dois ou mais antecedentes familiares estavam presentes.
Na Tabela 3 estão apresentados os resultados do odds ratio calculados comparando-se sobrepeso/obesidade (IMC maior ou igual a P85) com IMC normal (IMC entre P5 e P84). Foram analisadas as variáveis: sexo e antecedentes para DCV, verificando-se que os meninos tiveram (OR=1,37; IC 95\% 1,02-1,83) mais chance de apresentar sobrepeso/obesidade, assim como a presença de história familiar de DCV esteve associada a essas alterações nutricionais $(\mathrm{OR}=1,66$; IC95\% 1,23-2,23).

\section{Discussão}

No presente estudo, observou-se uma frequência baixa de desnutrição $(4,1 \%)$ e baixa estatura $(5,4 \%)$, contrastando com elevada taxa de sobrepeso $(14,4 \%)$ e obesos $(13,3 \%)$.

Tabela 1. Estado nutricional dos escolares, segundo idade. Embu, São Paulo, 2006.

\begin{tabular}{|c|c|c|c|c|c|c|c|c|c|c|}
\hline \multirow[b]{3}{*}{ Idade (Anos) } & \multicolumn{10}{|c|}{ Estado Nutricional } \\
\hline & \multicolumn{2}{|c|}{ Baixo Peso } & \multicolumn{2}{|c|}{ Sobrepeso } & \multicolumn{2}{|c|}{ Obeso } & \multicolumn{2}{|c|}{ IMC Normal } & \multicolumn{2}{|c|}{ Total } \\
\hline & $\mathrm{N}$ & $(\%)$ & $\mathbf{N}$ & $(\%)$ & $\mathbf{N}$ & $(\%)$ & $\mathbf{N}$ & $(\%)$ & $\mathbf{N}$ & $(\%)$ \\
\hline $6+7$ & 03 & $(2,1)$ & 24 & $(16,8)$ & 23 & $(16,1)$ & 93 & $(65,0)$ & 143 & $(100,0)$ \\
\hline $7 \vdash 7,9$ & 08 & $(3,6)$ & 35 & $(15,6)$ & 35 & $(15,6)$ & 146 & $(65,2)$ & 224 & $(100,0)$ \\
\hline $8+8,9$ & 07 & $(3,1)$ & 29 & $(13,0)$ & 26 & $(11,7)$ & 161 & $(72,2)$ & 223 & $(100,0)$ \\
\hline $9+9,9$ & 10 & $(5,1)$ & 25 & $(12,6)$ & 31 & $(15,7)$ & 132 & $(66,7)$ & 198 & $(100,0)$ \\
\hline $10 \vdash$ & 10 & $(7,1)$ & 21 & $(14,9)$ & 09 & $(6,4)$ & 101 & $(71,6)$ & 141 & $(100,0)$ \\
\hline Total & 38 & $(4,1)$ & 134 & $(14,4)$ & 124 & $(13,3)$ & 633 & $(68,1)$ & 929 & $(100,0)$ \\
\hline
\end{tabular}

$\mathrm{p}=0,168$

Tabela 2. Estado nutricional dos escolares, segundo sexo, peso ao nascer e presença de antecedentes familiares para DCV. Embu, São Paulo, 2006.

\begin{tabular}{|c|c|c|c|c|c|c|c|c|c|c|c|}
\hline & \multicolumn{11}{|c|}{ Estado Nutricional } \\
\hline & \multicolumn{2}{|c|}{ Baixo Peso } & \multicolumn{2}{|c|}{ Sobrepeso } & \multicolumn{2}{|c|}{ Obeso } & \multicolumn{2}{|c|}{ IMC Normal } & \multicolumn{2}{|c|}{ Total } & \multirow[b]{2}{*}{$p$} \\
\hline & $\mathbf{N}$ & $(\%)$ & $\mathrm{N}$ & $(\%)$ & $\mathbf{N}$ & $(\%)$ & $\mathbf{N}$ & $(\%)$ & $\mathbf{N}$ & $(\%)$ & \\
\hline Sexo & & & & & & & & & & & $0,035^{*}$ \\
\hline Masculino & 23 & $(5,5)$ & 70 & $(16,6)$ & 60 & $(14,2)$ & 269 & $(63,7)$ & 422 & $(100,0)$ & \\
\hline Feminino & 15 & $(3,0)$ & 64 & $(12,6)$ & 64 & $(12,6)$ & 364 & $(71,8)$ & 507 & $(100,0)$ & \\
\hline Peso ao nascer ${ }^{1 * *}$ & & & & & & & & & & & 0,105 \\
\hline Baixo peso $<2500 \mathrm{~g}$ & 06 & $(7,9)$ & 05 & $(6,6)$ & 09 & $(11,8)$ & 56 & $(73,7)$ & 76 & $(100,0)$ & \\
\hline Peso insuficiente & 10 & $(5,9)$ & 28 & $(16,5)$ & 19 & $(11,2)$ & 113 & $(66,5)$ & 170 & $(100,0)$ & \\
\hline Adequado & 19 & $(3,6)$ & 80 & $(15,0)$ & 80 & $(15,0)$ & 356 & $(66,5)$ & 535 & $(100,0)$ & \\
\hline Macrossômico & 0 & $(0)$ & 10 & $(25,0)$ & 05 & $(12,5)$ & 25 & $(62,5)$ & 40 & $(100,0)$ & \\
\hline Ant. fam. p/DCV & & & & & & & & & & & $0,000^{*}$ \\
\hline Sim & 16 & $(4,9)$ & 40 & $(12,2)$ & 72 & $(22,0)$ & 200 & $(61,0)$ & 328 & $(100,0)$ & \\
\hline Não & 22 & $(3,7)$ & 94 & $(15,6)$ & 52 & $(8,7)$ & 433 & $(72,0)$ & 601 & $(100,0)$ & \\
\hline
\end{tabular}

*p<0,05 Teste Qui quadrado ** Sem informação=107 (11,6\%) ${ }^{1}$ : Classificação OMS 
Tabela 3. Sobrepeso e obesidade em escolares segundo sexo e antecedentes familiares para DCV. Embu, São Paulo, 2006.

\begin{tabular}{|c|c|c|c|c|c|}
\hline & \multicolumn{2}{|c|}{ Sobrepeso /Obeso } & \multirow[b]{2}{*}{$\mathrm{p}$} & \multirow{2}{*}{ Intervalo de confiança (95\%) } & \multirow{2}{*}{$\mathrm{p}$} \\
\hline & $\mathbf{N}$ & $(\%)$ & & & \\
\hline \multicolumn{6}{|l|}{ Sexo } \\
\hline Masculino & 130 & $(32,6)$ & 1,37 & $1,02-1,83$ & 0,032 \\
\hline Feminino & 128 & $(25,2)$ & & & \\
\hline \multicolumn{6}{|c|}{ Ant. fam. p/DCV } \\
\hline Sim & 112 & $(35,9)$ & 1,66 & $1,23-2,23$ & 0,001 \\
\hline Não & 146 & $(25,2)$ & & & \\
\hline
\end{tabular}

Este resultado - prevalência de obesidade - é semelhante ao encontrado em crianças de 6-11 anos nos EUA $(18,8 \%)$ e acima de descrições para essa faixa etária em países como a França $(6 \%)^{29-31}$. Braunschweig et al. ${ }^{32}$ avaliaram escolares de origem afro-americana, com idade média de 10 anos em escolas públicas de Chicago e observaram 23\% de obesos (IMC > P90). Em estudo entre crianças (7-10 anos) de escolas públicas no município de Santos (SP), Costa et al. ${ }^{33}$ observaram obesidade em 16,8 e 14,3\%, respectivamente, entre meninos e meninas; resultados semelhantes foram obtidos por Suñé et al. ${ }^{34}$ que relataram prevalência de 24,8\% de sobrepeso/obesidade em escolares de uma cidade na região sul do Brasil.

A história familiar de risco de DCV foi positiva em 35,3\% dos escolares, sendo que o antecedente mais frequentemente relatado foi a DCV precoce pais/avós (20\%). Embora as informações tenham sido colhidas de questionário sem confirmação clínica, o achado de pelo menos um antecedente familiar: obesidade, hipertensão arterial, diabetes mellitus, dislipidemia e DCV precoce em mais de um terço dos pais/avós dessas crianças reiteram a importância da avaliação desse fator de risco - história familiar - tanto para antecipação da identificação de crianças/adolescentes de risco, como pela oportunidade de orientação visando a família como um todo, ou seja, orientações alimentares, de atividades físicas, de hábitos de vida mais saudáveis para as crianças e para seus pais e/ou responsáveis. A associação significante entre a variável sobrepeso/obesidade e a presença de antecedentes familiares para DCV nos escolares, demonstra a complexidade e o sinergismo de fatores genéticos e ambientais na etiologia dessa doença.

O estudo de Reis et al. ${ }^{35}$ demonstrou que entre crianças obesas há um risco cinco vezes maior dos pais serem obesos. Como as crianças frequentam mais comumente serviços de atenção básica do que os adultos, estas poderiam servir como caso índice para risco aumentado em suas famílias. Os autores sugerem uma triagem universal para fatores de risco DCV para as crianças, o que proveria uma oportunidade para intervenção nas crianças e em seus pais, proporcionando maiores benefícios individuais e coletivos. A determinação de fatores de risco DCV em crianças pode identificar risco aumentado de DCV em seus pais.

O baixo peso ao nascer, considerado como um indicador de saúde pública, foi referido em $9,4 \%$ dos escolares, valores próximos a dois inquéritos domiciliares realizados na população infantil da cidade de São Paulo (1995/1996) e no Embu (1996), nos quais observaram-se prevalências de crianças nascidas com baixo peso de $8,9 \%$ e $8,4 \%$, respectivamente ${ }^{36,37}$. Entretanto, tem se verificado que, embora os índices de baixo peso ao nascer possam ser semelhantes em regiões mais ou menos desenvolvidas, a determinação dessa condição pode ser diversa. Em regiões mais desenvolvidas o baixo peso ao nascer relacionase sobretudo às gestações de risco como hipertensão arterial, diabetes, maior idade materna e gestações múltiplas por reprodução assistida; em países em desenvolvimento, resulta principalmente de restrição de crescimento intrauterino consequente à desnutrição materna, infecções congênitas, à falta de acesso e qualidade do prénatal para abordagem oportuna de intercorrências desse período e, ainda, excesso de indicações de cesarianas ${ }^{38}$. Com frequência, sobretudo em grandes centros urbanos, há concomitância dessas condições constituindo o baixo peso ao nascer importante critério de risco para acompanhamento e definição de ações diferenciadas para essas crianças.

O estudo de Silveira et al. ${ }^{39}$, em adolescentes de escolas públicas em São Paulo, não observou associação significante entre peso ao nascer e so- 
brepeso/obesidade. No presente estudo, essa associação também não foi observada, porém, constatou-se $18,4 \%$ de sobrepeso/obesidade nas crianças nascidas com peso inferior a $2500 \mathrm{~g}$, o que poderia ser indicativo de uma possível evolução para mudança no padrão nutricional das mesmas. A impossibilidade de confirmação da existência de restrição de crescimento intrauterino, neste estudo, impediu a análise desse fator com o estado nutricional atual das crianças participantes.

Há poucos estudos sobre a programação fetal em países em desenvolvimento, pois estes dependem da manutenção de arquivos do peso ao nascer para serem comparados com a saúde dos adultos ${ }^{40}$. Entretanto, as estimativas apontam que a maioria das crianças que apresentou restrição de crescimento intrauterino nasce em países em desenvolvimento, nos quais, simultaneamente, há a previsão de uma pandemia de obesidade e diabetes tipo 2. Assim, nesses países e regiões que sofrem com "double burden", ainda não totalmente controlada, fica clara a necessidade de implementação de uma agenda de doenças infecciosas associada a uma agenda emergente de doenças não transmissíveis com uma urgente readequação nos programas de saúde pública ${ }^{40,41}$.

Diante da alta prevalência de sobrepeso e obesidade em crianças pré-púberes observada nesta pesquisa, ou seja, 27,7\% com IMC $\geq$ P85, destaca-se a importância de uma atuação intersetorial, envolvendo principalmente as áreas da saúde e da educação. Em relação mais especificamente à área da educação, compreende-se que, na escola, diferentes ações podem ser desencadeadas - a atenção especial para não reproduzir e reforçar alimentações inadequadas, incentivar e possibilitar a prática de atividades físicas, assim como tornar-se um local de referência, junto aos alunos e suas famílias, para orientações de hábitos saudáveis. Nesse sentido, merece destaque o papel do Programa Nacional de Alimentação Escolar em nosso país ${ }^{42}$. Depois de um longo período que se inicia na década de 1930, de cunho predominantemente assistencialista, a Constituição de 1988 estabelece que a alimentação escolar é um direito constitucional. Desde então, verificaram-se mudanças significativas na sua organização, objetivos e gerenciamento, dentre eles o processo de descentralização para estados e municípios, criação dos conselhos de alimentação escolar (CAE) e desenvolvimento de ações e destinação de recursos diferenciados para alunos de creches, ensino fundamental, indígenas e quilombolas. Gerenciado pelo Fundo Nacional de Desenvolvimento da Educação (FNDE), desde 1998, o Programa Nacional de Alimentação Escolar tem desenvolvido inúmeras ações de forma articulada. Um ponto a ser destacado refere-se à determinação prevista nessa legislação de se oferecer ao aluno durante sua permanência na escola pelo menos uma refeição que garanta 30\% das necessidades nutricionais diárias, apontando dentre os objetivos a promoção do crescimento e desenvolvimento e de melhor aprendizagem e rendimento escolar ${ }^{42,43}$. Denota-se, assim, uma preocupação prioritária com os déficits nutricionais, visão esta que precisa ser reavaliada em função das mudanças observadas no perfil nutricional da população pediátrica, sobretudo nos grandes centros urbanos.

Em conclusão, os achados deste estudo apontaram para o aumento da prevalência do excesso de peso nessa faixa etária, sugerindo semelhanças com países desenvolvidos e, ainda, associação dessa condição com os antecedentes familiares positivos para DCV. Há, portanto, urgência em redirecionar os esforços de programas e recursos para a melhoria da condição nutricional, visando a prevenção e o controle da obesidade nessa faixa etária. Esses resultados indicam os possíveis benefícios da detecção dessas condições em uma população de escolares, incluindo estratégias de orientação para prevenção da obesidade nas escolares e orientação dos familiares, além do tratamento para aquelas com doenças crônicas já instaladas.

\section{Colaboradores}

MWL Strufaldi e RF Puccini trabalharam na concepção e na redação final e EMK Silva, na metodologia e análise na estatística. Todos os autores participaram da concepção e análise dos dados. 


\section{Referências}

1. De Onis M, Blossner M, Borghi E, Morros R, Frongillo EA. Methodology for estimating regional and global trends of child malnutrition. Int J Epidemiol 2004; 33(6):1260-1270.

2. Brasil. Ministério da Saúde (MS). Pesquisa Nacional Demografia e Saúde da Criança e da Mulher. PNDS. Brasília: Ministério da Saúde (MS); 2006.

3. Drewnowskil A. Nutritional transition and global dietary trends. Nutrition 2000;16(7-8):486-487.

4. Monteiro CA, Conde WL, Popkin BM. Independent effects of income and education on the risk of obesity in the brazilian adult population. J Nutr 2001; 131(3):881S-886S.

5. Organización Panamericana de la Salud. La Obesidad en la Pobreza: Un Nuevo Reto para la Salud Pública. 2000; Publicación Científica 576. Washington, DC: OPS.

6. Flynn MA, McNeil DA, Maloff B, Mutasingwa D, Wu M, Ford C, Tough SC. Reducing obesity and related chronic disease risk in children and youth: a synthesis of evidence with 'best practice' recommendations. Obes Rev 2006; 7(Supl. 1):7-66.

7. Batista Filho M, Rissin A. A transição nutricional no Brasil: tendências regionais e temporais. Cad Saude Publica 2003; 19(Supl. 1):181-191.

8. Gigante DP. Avaliação nutricional de adultos da coorte de nascimentos de 1982, Pelotas (RS). Rev Saude Publica 2008; 42(Supl.2):60-69.

9. Barros AJD, Victora CG, Santos IS, Matijasevich A, Araújo CL, Barros FC. Infant malnutrition and obesity in three population-based birth cohort studies in Southern Brazil: trends and differences. Cad Saude Publica 2008; 24(Supl. 3):S417-S426.

10. Batista Filho M, Souza AI, Miglioli TC, dos Santos MC. Anemia e obesidade; um paradoxo da transição nutricional brasileira. Cad Saude Publica 2008; 24(Supl. 2):S247-S257.

11. Instituto Brasileiro de Geografia e Estatística (IBGE). Pesquisa de Orçamentos Familiares 2002/2003. Antropometria e análise do estado nutricional de crianças $e$ adolescentes no Brasil. Rio de Janeiro: Instituto Brasileiro de Geografia e Estatística (IBGE); 2006.

12. Marins VMR, Almeida RMVR, Pereira RA, Barros MBA. The relationship between parental nutritional status and overweight children/adolescents in Rio de Janeiro, Brazil. Public Health 2004; 118(1):43-49.

13. Mello ED, Luft VC, Meyer F. Obesidade infantil: como podemos ser eficazes? J Pediatr 2004; 80(3): 173-182.

14. Sotelo YOM, Colugnati FAB, Taddei JAAC. Prevalência de sobrepeso e obesidade entre escolares da rede pública segundo três critérios de diagnóstico antropométrico. Cad Saude Publica 2004; 20(1):233240.

15. Aregullin-Eligio EO, Alcorta-Garza MC. Prevalencia y factores de riesgo de hypertension arterial en escolares mexicanos: caso Sabinas Hidalgo. Salud Pública Méx 2009; 51(1):14-18.

16. Araújo TL, Lopes MVO, Cavalcante TF, Guedes NG, Moreira RP, Chaves ES,Silva VM. Análise de indicadores de risco para hipertensão arterial em crianças e adolescents. Rev Esc Enferm USP 2008; 42(1):120-126.
17. Nogueira PCK, Costa RF, Cunha JSN, Silvestrini L, Fisberg M. Pressão arterial elevada em escolares de Santos: relação com obesidade. Rev Assoc Med Bras 2007; 53(5):426-432.

18. Guimarães ICB, Guimarães AC. Síndrome metabólica na infância e adolescência: um fator maior de risco cardiovascular. Rev Baiana Saúde Pública 2006; 30(2):349-362.

19. Phenekos C. Influence of fetal body weight on metabolic complications in adult life: review of the evidence. J Pediatr Endocrinol Metab 2001; 14(Supl. 5):1361-1363.

20. Levy-Marchal C, Jaquet D, Czernichow P. Long-term metabolic consequences of being born small for gestacional age. Semin Neonatol 2004; 9(1):67-74.

21. Da Silveira VML, Horta BL. Peso ao nascer e síndrome metabólica em adultos: meta análise. Rev Saude Publica 2008; 42(1):10-18.

22. Clayton PES, Cianfarani S, Czernichow P, Johannsson G, Rapaport R, Rogol A. Management of the Child Born Small for Gestational Age Child (SGA) through to Adulthood: A Consensus Statement of the International Societies of Paediatric Endocrinology and the Growth Hormone Research Society. J Clin Endocrinol Metab 2007; 92(3):804-810.

23. Fundação Sistema Estadual de Análise de Dados (SEADE). Informações dos municípios paulistas. [acessado 2009 jun 4]. Disponível em: http:// www.seade.gov.br

24. Dean AG, Dean JA, Coloumber D, Burton AH, Brendel KA, Smith DC, Dicker RC, Sullivan KM, Fagan RF. Epi Info, Version 6: A word-processing, database, and statistics program for public health on IBM- compatible microcomputers. Centers for Disease Control and Prevention. Atlanta, Georgia USA, 1995.

25. World Health Organization (WHO). International Statistical Classification of disease and related health problems; tenth revision. Geneva: WHO; 1992.

26. Williams CL, Hayman LL, Daniels SR, Robinson TN, Steinberger J, Paridon S, Bazzarre T. Cardiovascular Health in Childhood A Statement for Health Professionals From the Committee on Atherosclerosis, Hypertension, and Obesity in the Young (AHOY) of the Council on Cardiovascular Disease in the Young, American Heart Association. Circulation 2002; 106(1):143-160.

27. Kuczmarski RJ, Ogden CL, Grummer-Strawn LM, Flegal KM, Guo SS, Wei R, Curtin LR, Roche AF, Johnson CL. CDC growth charts: United States. Adv Data 2000; 314:1-28.

28. Speiser PW, Rudolf MCJ, Anhalt H, Camacho-Hubner C, Chiarelli F, Eliakim A, Freemark M, Gruters A, Hershkovitz E, Iughetti L, Krude H, Latzer Y, Lustig RH, Pescovitz OH, Pinhas-Hamiel O, Rogol AD, Shalitin S, Sultan C, Stein D, Vardi P, Werther GA, Zadik Z, Zuckerman-Levin N, Hochberg Z. Consensus Statement: Childhood Obesity. J Clin Endocrinol Metab 2005; 90(3):1871-1887. 
29. Centers for Disease Control and Prevention, National Center for Health Statistics. NHANES (1982-84) and NHANES (1963-65 and 1966-70), United States, 2003. Overweight children and adolescents 6-19 years of age, according to sex, age, race and hispanic origin: United States, selected years 1963-65 through 1999-2000. [acessado 2007 dez 10]. Disponível em: http://www.cdc.gov/nchs/products/pubs/pubd/ hestats/overwght99.htm

30. Ogden CL, Carroll MD, Curtin LR, McDowell MA, Tabak CJ, Flegal KM. Prevalence of Overweight and Obesity n the United States, 1999-2004. JAMA 2006; 295(13):1549-1555.

31. Rolland-Cachera MF, Castetbon K, Arnault N, Bellisle F, Romano MC, Lehingue Y, Frelut ML, Hercberg S. Body mass index in 7-9-y-old French children: frequency of obesity, overweight and thinness. Int J Obes Rel Metab Disord 2002; 26(12):1610-1616.

32. Braunschweig CL, Gómez S, Liang H, Tomey K, Doerfler B, Wang Y, Beebe C, Lipton R. Obesity and risk factors for the metabolic syndrome among lowincome, urban, African American schoolchildren: the rule rather than the exception? Am J Clin Nutr 2005; 81(5):970-975.

33. Costa RF, Cintra IP, Fisberg M. Prevalence of Overweight and Obesity in School Children of Santos City,Brazil. Arq Bras Endoc Metab 2006; 50(1):60-67.

34. Suñè FR, Da Costa JSD, Olinto MTA, Pattussi MP. Prevalência e fatores associados para sobrepeso e obesidade em escolares de uma cidade no Sul do Brasil. Cad Saude Publica 2007; 23(6):1361-1371.

35. Reis EC, Kip KE, Marroquin OC, Kiesau M, Hipps L, Jr, Peters RE, Reis SE. Screening Children to Identify Families at Increased Risk for Cardiovascular Disease. Pediatrics 2006; 118(6):1789-1797.

36. Monteiro CA, Benício MHD, Ortiz LP, Conde WL. Tendência secular do peso ao nascer na cidade de São Paulo (1976-1998). Rev Saude Publica 2000; 34(Supl. 6):26-40.

37. Puccini RF, Silva NN, Araújo NS, Pedroso GC, Silva EMK. Saúde infantil: condições de vida e utilização de serviços de saúde em área da Região Metropolitana de São Paulo, 1996. Rev bras saúde matern infant 2002; 2(2):143-155.
38. Villar J, Valladares E, Wojdyla D, Zavaleta N, Carroli G, Velazco A, Shah A, Campodónico L, Bataglia V, Faundes A, Langer A, Narváez A, Donner A, Romero M, Reynoso S, de Pádua KS, Giordano D, Kublickas M, Acosta A. Caesarean delivery rates and pregnancy outcomes: the 2005 WHO global survey on maternal and perinatal health in Latin América. Lancet 2006; 367(9525):1819-1829.

39. Silveira D, Taddei JAAC, Escrivão MAMS, Oliveira FLC, Ancona-Lopez F. Risk factors for overweight among Brazilian adolescents of low-income families: a case control study. Public Health Nutr 2006; 9(4):421-428

40. Prentice AM, Moore SE. Early programming of adult diseases in resource poor Countries. Arch Dis Child 2005; 90(4):429-432.

41. De Onis M, Blossner M, Villar J. Levels and patterns of intrauterine growth retardation in developing countries. Eur J Clin Nutr 1998; 52(Supl. 1):5-15.

42. Brasil. Ministério da Educação (MEC). Programa Nacional de Alimentação Escolar (PNAE). Resolução no 33 de 24 de agosto de 2006. [acessado 2009 jun 4]. Disponível em www.fnde.gov.br

43. Brasil. Ministério da Educação (MEC). Fundo Nacional de desenvolvimento da Educação. Conselho Deliberativo. Resolução/CD/FNDE No 38 de 16 de julho de 2009. [acessado 2009 ago 30]. Disponível em: ftp://ftp.fnde.gov.br/web/resolucoes_2009/ res038_16072009.pdf

Artigo apresentado em 26/06/2009

Aprovado em 04/09/2009

Versão final apresentada em 15/09/2009 